\title{
Service de garde: tout le monde en veut, mais qui doit payer?
}

\section{Remo Osterwalder}

Dr med., vice-président de la FMH, responsable du département Sauvetage

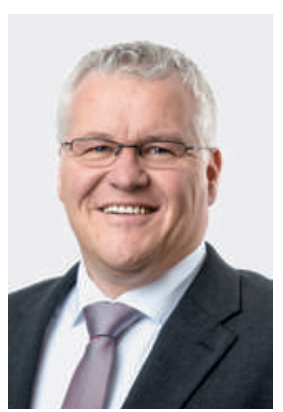

Qui n'a pas connu ça: l'ouverture du cabinet approche après un long dédale administratif. Mais avant, les collègues de la société médicale cantonale en profitent pour nous rappeler non seulement les droits d'un médecin indépendant, mais surtout ses devoirs. En d'autres termes, on est accueilli à bras ouverts dans le service de garde, qui est toujours un devoir dans la plupart des régions. Un devoir que nous avons déjà exercé au cours de notre formation de spécialiste dans les services d'urgence des hôpitaux, mais un devoir aussi qui, le plaisir mis à part, montre le revers de la médaille de cette prestation du système de santé. Ces services sont peut-être attrayants en début de carrière, quand on vient de s'installer, car quand un patient n'a pas encore de médecin de famille, il peut le trouver par ce biais. Mais en réalité, il arrive malheureusement bien souvent qu'une partie de ces consultations d'urgence restent impayées. En effet, certains patients pensent que cette offre est mise à leur disposition par l'Etat ou, plus généralement, ils éprouvent des difficultés à répondre aux sollicitations administratives que cela implique.

\section{Le service de garde est une prestation essen-} tielle au service de la population.

L'organisation du service de garde est aussi multiple et hétérogène que la carte de la Suisse. Aujourd'hui déjà, dans plusieurs régions, le service de garde ne peut plus être garanti par les médecins de premier recours locaux, en particulier lorsque ceux-ci atteignent l'âge de la retraite. Il est possible de pallier certaines situations critiques par une coopération avec les hôpitaux de proximité (soins généraux). Mais le problème principal de ces institutions réside dans le fait qu'elles ne sont pas très mobiles et par conséquent qu'elles ne peuvent souvent pas dispenser de traitements hors muros, par exemple sous forme de visites à domicile. Si les régions rurales sont plus particulièrement touchées, la question se pose quand même de manière générale: comment augmenter l'attractivité des interventions d'ur- gence pour les jeunes collègues? On ne peut pas faire comme si c'était dans l'ADN de l'homo medicus de passer ses week-ends loin de sa famille par pur plaisir. Non seulement les attentes des jeunes générations de médecins changent, mais aussi celles, légitimes, de leur famille.

En principe, la protection et la promotion de la santé de la population, urgences incluses, relèvent des cantons, qui les garantissent dans une mesure limitée par leurs institutions cantonales, je dis limitée, car les visites à domicile et les consultations de proximité en font

En ambulatoire, la rémunération des prestations d'urgence devrait aussi se faire selon le principe des prestations d'intérêt général.

plutôt rarement partie. Les prestations d'urgence des hôpitaux sont rémunérées avec l'aide des prestations d'intérêt général, financées par la communauté (contributions cantonales), ce qui est en revanche rarement le cas pour les collègues médecins installés. Du point de vue des cantons, il devrait y avoir ici aussi un intérêt à rémunérer les prestations d'urgence des médecins indépendants selon le même principe. Tout comme le devoir légal de participer au service de garde existe dans tous les 26 cantons, cette mesure pourrait constituer le fondement de la rémunération et de la revalorisation de ce service, tout en apportant un avantage à la population. De leurs côtés, les médecins ont déjà ouvert la voie et revalorisé cette prestation, par exemple avec le cours pour les médecins de garde de la Société suisse de médecine d'urgence et de sauvetage (SSMUS) ou avec le nouveau cursus de médecine d'urgence pour les étudiants en médecine de l'Université de Bâle en collaboration avec la FMH et la SSMUS. Pour le corps médical, la qualité est prioritaire, mais elle va de pair avec une reconnaissance et une amélioration proactive de l'attractivité du service de garde - et ce avant que des lacunes apparaissent à grande échelle dans la couverture de ce service. 